\title{
Slow Light Enabled Wavelength Demultiplexing
}

\author{
Z. Hayran ${ }^{1}$, M. Turduev, ${ }^{2}$ M. Botey, ${ }^{3}$ R. Herrero, ${ }^{3}$ K. Staliunas, ${ }^{3,4}$ and H. Kurt ${ }^{1}$ \\ ${ }^{I}$ Nanophotonics Research Laboratory, Department of Electrical and Electronics Engineering, TOBB University \\ of Economics and Technology, Ankara 06560, Turkey \\ ${ }^{2}$ Department of Electrical and Electronics Engineering, TED University, Ankara 06420, Turkey \\ ${ }^{3}$ DONLL, Departament de Física, Universitat Politècnica de Catalunya (UPC), \\ Edifici Gaia, Rambla Sant Nebridi 22, 08222 Terrassa, Spain \\ ${ }^{4}$ Institució Catalana de Recerca i Estudis Avancats (ICREA), \\ Passeig Lluís Companys 23, 08010 Barcelona, Spain
}

\begin{abstract}
Photonic crystal waveguides supporting band gap guided modes hold great potential to tailor the group velocity of propagating light. We propose and explore different wavelength demultiplexer design approaches that utilize slow light concept. By altering the dielectric filling factors of each waveguide segment, one can show that different frequencies can be separated and extracted at different locations along the cascaded waveguide. Furthermore, to eliminate the inherent reflection loss of such a design, a composite structure involving a tapered waveguide with a side-coupled resonator is also presented. Such a structure features not only a forward propagating wave but also a backward propagating wave acting as a feedback mechanism for the drop channels. We show that by careful design of the waveguide and the resonator, the destructive and instructive interference of these waves can effectively eliminate the reflection loss and increase the coupling efficiency, respectively. Numerical and experimental verification of the proposed structures show that the targeted frequencies can be coupled out with low cross-talks and moderate quality factors, while maintaining a compact size.
\end{abstract}

Keywords: slow light, wavelength selective devices, channelled optical waveguides, photonic crystals.

\section{INTRODUCTION}

The idea of merging nanoscale photonic systems with slow light concept has attracted great attention, since it enables additional control over signal and energy transmission and compression in space. All-optical data processing [1,2], optical nonlinearity [3], optical storage [4], and quantum information processing [5] are some of the key topics, that have benefited from the slow light enhanced light-matter interactions. Material and structural dispersion are of the two uttermost important factors that lie in the heart of any slow light devices. While slow light effect based on material dispersion arises from the change in the material index, due to wavelength dependent dipole response of the medium; the latter is enabled by unique dispersion characteristics of engineered media, such as photonic crystals (PCs) and dispersion compensating fibers. The feasible on-chip integration and freedom of operational bandwidth selection of PCs made these artificial structures especially attractive for slow light studies [6-8]. PCs with single or multiple omitted lines of rods, namely PC waveguides, are one of the most common configurations to achieve slow light effect at room temperatures. By careful design of the PC waveguide mode(s) dispersion properties, one can obtain frequency ranges where the group index experienced by the propagating wave is so high that the group velocity of the wave approaches nearly to zero. However, since these frequencies typically lie in the vicinity of the Brillouin zone boundaries, slow light achievement in PCs is generally associated with high radiation losses and high dispersion [9]. The first issue can be eliminated by designing a flat region in the band structure that lies below the so-called light line, whereas the latter issue requires special examination.

A light entering the slow light regime will be dispersed in space due to high frequency-group index dependency, leading to a distorted optical signal. To solve this issue, for instance a dispersion compensated slow light device is proposed and experimentally realized [10], where a positive dispersion in the first part of the structure is cancelled by a negative dispersion in the second part of the structure. Another method employs straightening the guided-mode band, so that dispersion becomes effectively zero [11]. While it seems that the wave dispersion in space is an undesirable phenomenon, this effect can in fact be exploited to synchronously extract narrowband signals at drop channels, which can be placed at different locations along the slow light supporting main waveguide. As schematically depicted in Fig. 1(a), in wavelength demultiplexers (DEMUXs) operating in a linear dispersion region [12,13], signals with different frequencies will arrive at its regarding arbitrary positioned channel position at different times, since the linear dispersion regime dictates a constant group velocity, leading to asynchronous output signals at the drop channels. On the other hand, in a DEMUX where the main and/or the drop waveguide are systematically slow light engineered, a natural drop channel position can occur for each frequency, so that the narrowband frequencies enter and leave the drop channel nearly synchronized [see Fig. 1(b)], if the minor channel position mismatches imposed by the discreteness of the crystal structure and the possible dispersion in the drop channels are omitted. In other words, the slow light device acts as a natural optical delay line [1,2]. In this study, we present numerically and experimentally two DEMUX designs, where the main channel is carefully designed to support slow light regime, leading to spatial 
separation of different frequencies. The first structure (DEMUX-1) consists of multiple segments of PC waveguides with different filling factors [14]. The cut-off frequency variation of these segments provides spatial confinement for the target frequencies, which can be then guided through drop channels. The second structure (DEMUX-2) [15] consists of a PC waveguide with a continuously changing waveguide width, which results in light trapping and reflection at particular positions. Furthermore, the adiabatically tapered waveguide is accompanied by side-coupled PC cavities which are responsible for light extraction from both the incoming signal and reflected signal.
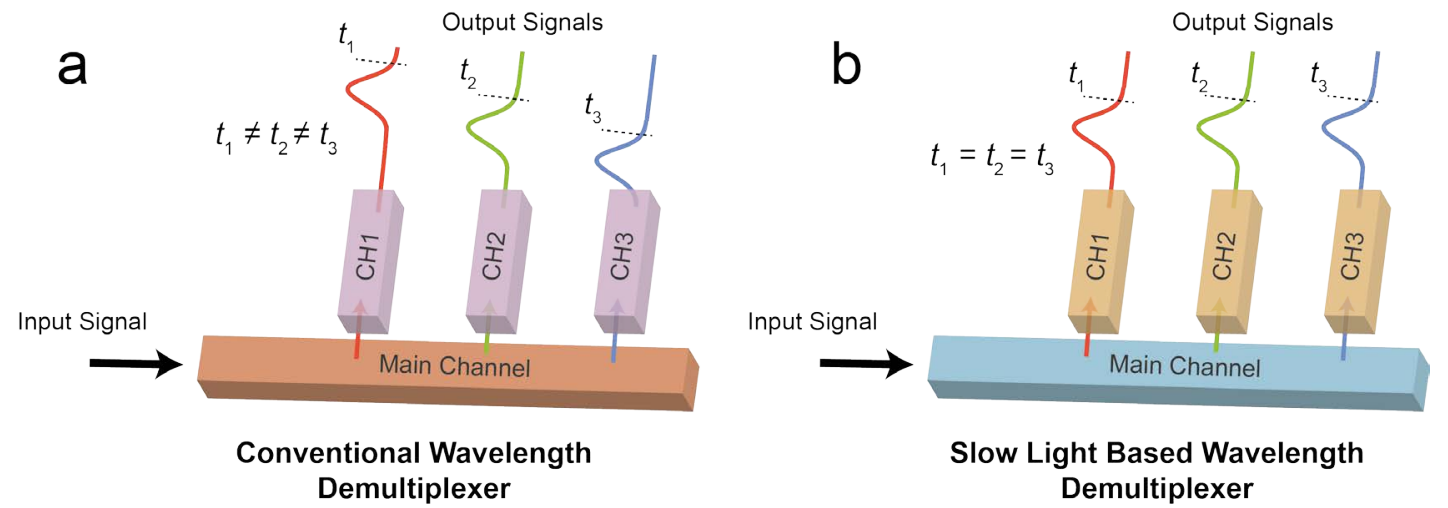

Figure 1. General schematics for a DEMUX design based on (a) linear guided-mode dispersion and (b) slow light effect are shown. The slow light effect can naturally synchronize the separated frequencies in time, thus eliminating the need of an optical delay line.

In the next section, we present two different DEMUX configurations based on two-dimensional PCs.

\section{WAVELENGTH DEMULTIPLEXER DESIGNS}

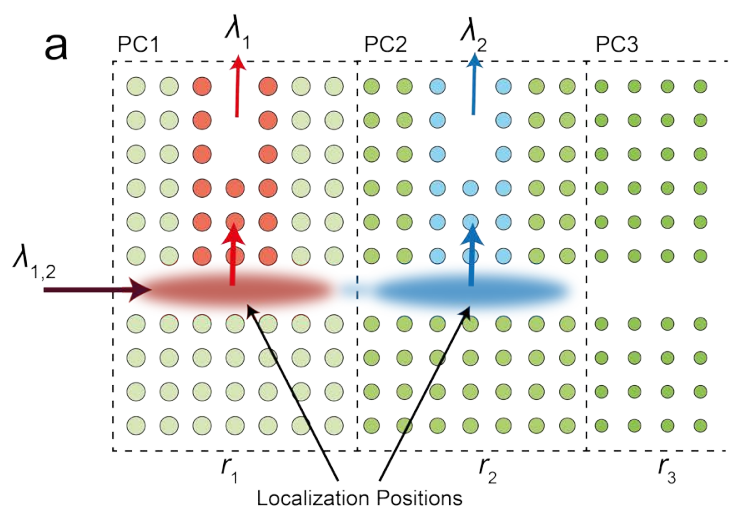

DEMUX-1

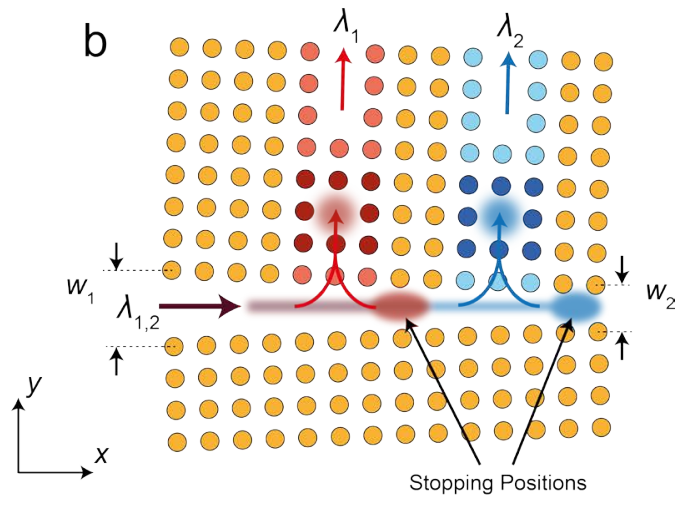

DEMUX-2

Figure 2. Schematic descriptions of the (a) DEMUX-1 and (b) DEMUX-2 designs are shown. The dashed lines outline the different $P C$ regions with different filing factors in (a). The red and blue colored rods show the drop channel regions in (a) and (b).

Figures 2(a) and 2(b) schematically illustrate the DEMUX-1 and DEMUX-2 designs, respectively. As can be seen from Fig. 2(a), the DEMUX-1 design consists of three cascaded PC waveguide regions with different filling factors, but in principle the design can be extended to any number of cascaded regions. The different filling factors can be achieved in numerous ways, such as varying the radii of the rods or changing the lattice constant of the regions. Here, as a basic demonstration three PC regions with lattice periods equal to $a_{1}=0.878 \mathrm{~cm}$, $a_{2}=0.790 \mathrm{~cm}$ and $a_{3}=0.718 \mathrm{~cm}$ are employed, respectively. The difference in the lattice period leads to local band gaps for specific frequencies, due to the frequency shift at the guide-mode. The PC rods are made of Alumina $\left(\mathrm{Al}_{2} \mathrm{O}_{3}\right)$ which has a dielectric constant equal to 9.61 at microwave frequencies. The radii and the height of the rods are equal to $r_{1}=0.158 \mathrm{~cm}$ and $h_{1}=15.32 \mathrm{~cm}$, respectively. The specific frequency range that lies between cut-off frequencies of the two consecutive PC regions is then localized at the regarding section. Due to its low group velocity the localized wave penetrates more into the surrounding PC cladding than the nonlocalized waves, and therefore can be easily leaked out if one introduces drop channels that are transverse to the propagation direction in the main channel. 
On the other hand, as illustrated in Fig. 2(b), the DEMUX-2 design is based on a tapered PC waveguide with side coupled defect-based cavities. In contrast to the first design, the DEMUX-2 has a smooth cut-off frequency variation along the propagation $x$-direction. This property results in a gradually increasing spatial period along the propagation direction, which relaxes the phase condition required for a high transmission. This phase condition can be analytically predicted using the well-known coupled mode analysis. By using time-reversal symmetry and power conservation principle, the transmission spectrum for a single channel can be derived as follows,

$$
|T(\omega)|=\left|\frac{\sqrt{\frac{4}{\tau_{3}}}\left(\sqrt{\frac{1}{\tau_{1}}}+\sqrt{\frac{1}{\tau_{2}}} e^{-i \phi(\omega)}\right)}{i\left(\omega-\omega_{0}\right)+\frac{1}{\tau_{1}}+\frac{1}{\tau_{3}}+\frac{1}{\tau_{2}}\left(1+2 e^{-i \phi(\omega)}\right)}\right|^{2},
$$

where $1 / \tau_{1}, 1 / \tau_{2}$ and $1 / \tau_{3}$ denote the decay rates occurring due to leakages into the input channel, reflection channel and the drop channel, respectively. On the other hand, $\omega_{0}$ is the resonance frequency of the PC cavity, and $\varphi(\omega)$ is the phase retardation due to the reflection inside the main channel. This analytical approach predicts that the PC cavity should be placed at positions where the phase delay for the resonant wavelength is an integer multiply of $2 \pi$. In such a case, the reflection occurring due to the cut-off variation can interfere with the incoming signal constructively and couple to specifically tuned PC cavities, leading to high transmission. The designed PC structure consists of $\mathrm{Al}_{2} \mathrm{O}_{3}$ rods with a radius and height equal to $r_{1}=0.317 \mathrm{~cm}$ and $h_{1}=15.50 \mathrm{~cm}$, respectively. The lattice constant is equal to $a=1.00 \mathrm{~cm}$, whereas the width of the front and end side of the waveguide are equal to $w_{1}=1.98 \mathrm{~cm}$ and $w_{2}=1.42 \mathrm{~cm}$, respectively.

The proposed designs were numerically verified using the finite-difference time-domain (FDTD) method [16] and experimentally realized in the microwave regime. The experimental setup consists of a vector network analyzer (Agilent HP-8510C for DEMUX-1 and E5071C ENA for DEMUX-2) and two standard horn antennas. Moreover, the setup was covered with microwave absorbers to build an anechoic chamber.

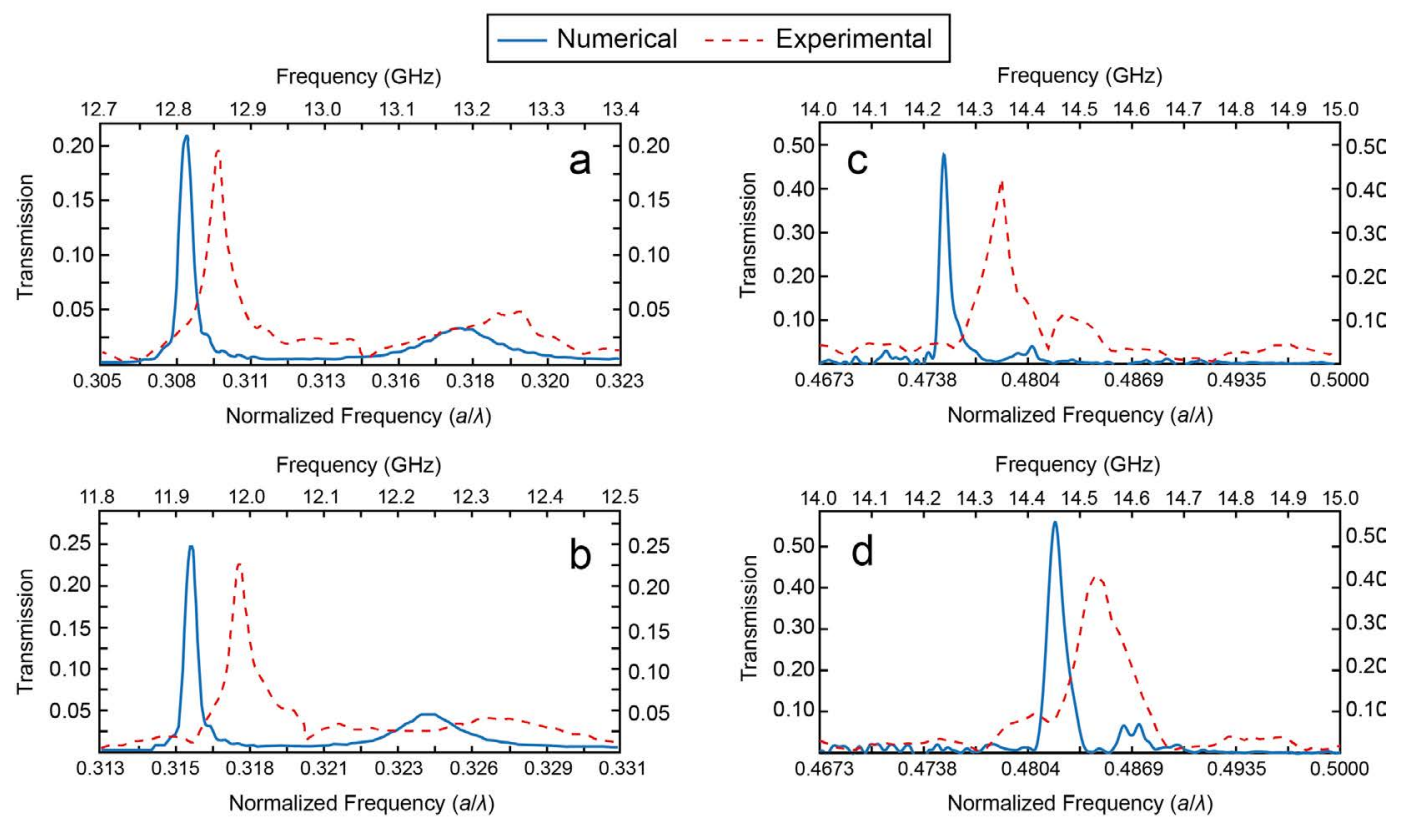

Figure 3. Calculated and experimentally measured transmission spectra for the (a-b) DEMUX-1 and (c-d) $D E M U X-2$ designs are shown, obtained from the $(a-c)$ first and $(b-d)$ second channel.

Figures 3(a-b) and 3(c-d) show the numerically calculated and experimentally measured transmission spectra for the DEMUX-1 and DEMUX-2 design, respectively. It can be seen from these figures, that the reflection feedback greatly enhances the transmission efficiency in comparison with the DEMUX-1 design. Furthermore, one can observe a slight shift in the frequency, which may be due to fabrication/material imperfections and effective index variation imposed by the finite structural height. The cross-talks for DEMUX-1 are obtained as $-13.7 \mathrm{~dB}$ and $-15.6 \mathrm{~dB}$ for the first and second channel, respectively; and for DEMUX-2 as $-12.2 \mathrm{~dB}$ and $-7.3 \mathrm{~dB}$ for the first and second channel, respectively. 


\section{CONCLUSION}

In summary, by exploiting the unique features of the slow light phenomenon, we have proposed and realized two different DEMUX designs. Both designs take advantage of the low group velocity of light that increases the interaction between the frequency of interest and the drop channel. While the first design approach is to directly couple the localized wave into the drop channels, the second approach allows the localized wave to reflect and couple to side-coupled resonators by interfering with the incoming wave, thus increasing the transmission efficiency. We should note that the designed structures are relatively compact and feasible for nanofabrication. Furthermore, regarding the incorporation of slow light based DEMUX structures into integrated photonic devices, out-of-plane losses will be a key aspect, which need to be addressed. This can be either done by designing the waveguide mode so that its tangential components lie inside the light cone, or by utilizing three dimensional photonic band gap that confine light in all directions.

\section{ACKNOWLEDGEMENTS}

H.K. acknowledges the partial support of the Turkish Academy of Sciences.

\section{REFERENCES}

[1] D. Dahan and G. Eisenstein, "Tunable all optical delay via slow and fast light propagation in a Raman assisted fiber optical parametric amplifier: A route to all optical buffering," Opt. Express, 13(16), 6234$6249(2005)$.

[2] Y. Okawachi, M. Bigelow, J. Sharping, Z. Zhu, A. Schweinsberg, D. Gauthier, R. Boyd and A. Gaeta, "Tunable all-optical delays via Brillouin slow light in an optical fiber," Phys. Rev. Lett., 94(15), 153902 (2005).

[3] C. Monat, B. Corcoran, M. Ebnali-Heidari, C. Grillet, B. Eggleton, T. White, L. O'Faolain and T. Krauss, "Slow light enhancement of nonlinear effects in silicon engineered photonic crystal waveguides," Opt. Express, 17(4), 2944-2953 (2009).

[4] Y. Su, F. Liu, Q. Li, Z. Zhang and M. Qiu, "System performance of slow-light buffering and storage in silicon nano-waveguide," Asia-Pacific Opt. Communications, 67832-67832 (2007).

[5] M. Lukin and A. Imamoğlu, "Nonlinear optics and quantum entanglement of ultraslow single photons," Phys. Rev. Lett., 84(7), 1419-1422 (2000).

[6] Y. Vlasov, M. O'Boyle, H. Hamann and S. McNab, "Active control of slow light on a chip with photonic crystal waveguides," Nature, 438(7064), 65-69 (2005).

[7] J. Li, T. White, L. O'Faolain, A. Gomez-Iglesias and T. Krauss, "Systematic design of flat band slow light in photonic crystal waveguides," Opt. Express, 16(9), 6227-6232 (2008).

[8] H. Kurt, "All-dielectric periodic media engineered for slow light studies," International Journal of Modern Physics B 27, 1330020 (2013).

[9] T. Baba, "Slow light in photonic crystals," Nature Photonics, 2(8), 465-473 (2008).

[10] D. Mori, S. Kubo, H. Sasaki and T. Baba, "Experimental demonstration of wideband dispersioncompensated slow light by a chirped photonic crystal directional coupler," Opt. Express, 15(9), 5264-5270 (2007).

[11] A. Petrov and M. Eich, "Zero dispersion at small group velocities in photonic crystal waveguides," Appl. Phys. Lett., 85(21), 4866-4868 (2004).

[12] S. Fan, P. Villeneuve, J. Joannopoulos and H. Haus, "Channel drop filters in photonic crystals," Opt. Express, 3(1), 4-11 (1998).

[13] S. Kim, I. Park, H. Lim and C. Kee, "Highly efficient photonic crystal-based multichannel drop filters of three-port system with reflection feedback," Opt. Express, 12(22), 5518-5525 (2004).

[14] A. E. Akosman, M. Mutlu, H. Kurt, and E. Ozbay, "Compact wavelength de-multiplexer design using slow light regime of photonic crystal waveguides," Opt. Express, 19(24), 24129-24138 (2011).

[15] Z. Hayran, M. Turduev, M. Botey, R. Herrero, K. Staliunas and H. Kurt, "Numerical and experimental demonstration of a wavelength demultiplexer design by point-defect cavity coupled to a tapered photonic crystal waveguide," Opt. Letters, 41(1), 119-122 (2016).

[16] A. Oskooi, D. Roundy, M. Ibanescu, P. Bermel, J. Joannopoulos and S. Johnson, "Meep: A flexible freesoftware package for electromagnetic simulations by the FDTD method," Computer Physics Communications, 181(3), 687-702 (2010). 